\title{
On a hybrid Laplace-time domain approach to dynamic interaction problems
}

\author{
A. Nieto Ferro ${ }^{\mathrm{a}, \mathrm{b} *}$, D. Clouteau ${ }^{\mathrm{a}}$, N. Greffet ${ }^{\mathrm{b}}$ and G. Devésa ${ }^{\mathrm{b}}$ \\ ${ }^{a}$ LMSSMat (CNRS UMR 8579), École Centrale Paris, Grande Voie des Vignes, 92295 Châtenay- \\ Malabry, France; ${ }^{b}$ EDF R\&D (AMA Dept.)/LaMSID (CNRS UMR 2832), Avenue du Général de Gaulle \\ 1, 92141 Clamart, France
}

Dynamic interaction problems between two subdomains lead in time to convolution integrals on the interface when one (linear) subdomain is modelled by an impedance operator and the other exhibits non-linear behaviour. In the present work, a convolution quadrature method is used to address a Laplace transform-based approach to evaluate these convolution products. Its properties are discussed on some numerical soil-structure interaction applications: one that is fully linear and the other showing material non-linearities.

La résolution des problèmes dynamiques d'interaction entre deux sous-domaines dont l'un, linéaire, n'est connu que par une impédance de bord, entraîne, lorsqu'on cherche prendre en compte des non-linéarités, l'apparition d'intégrales de convolution dans le domaine temporel. Dans ce travail, on présente une approche pour les évaluer, basée sur la transformée de Laplace de la fonction d'impédance et une méthode de quadratures de convolution. Une analyse plus détaillée est ensuite poursuivie sur des cas pratiques d'interaction sol-structure dans un cadre linéaire et non-linéaire.

Keywords: impedance matrix; convolution quadrature method; dynamic soil-structure interaction

Mots-clés: matrice d'impédance; méthode de quadratures de convolution; interaction solstructure dynamique

\section{Introduction}

Dynamic interaction problems are often solved using a domain decomposition technique that involves two or more subdomains. In each of them a local boundary value problem is solved separately given prescribed boundary unknowns: a displacement fields in the primal approach. A weak form of the traction equilibrium on the interfaces between the subdomains provides the final equation to solve the problem. The approach addressed in this article is particularly interesting when one subdomain is fully linear and the other shows a non-linear behaviour. In this case, the effects of the linear subdomain on the interface can be represented by means of an impedance operator, assumed hereafter to be defined in the frequency or Laplace domain. The coupled problem is solved in the time domain on the non-linear subdomain with external forces applied on the interface accounting for the linear domain. These interaction forces

*Corresponding author. Email: alex.nieto-ferro@edf.fr 
involving the time form of the impedance operator lead to a convolution integral that has to be computed.

These convolution products are usually found when fluid-structure or soil-structure interaction (SSI) is taken into account. For the latter, literature proposes several approaches for the evaluation of the interaction forces such as the time-frequency method (Darbre \& Wolf, 1988 ) or the hidden variables method (Cottereau, Clouteau, Soize, \& Cambier, 2006) which, using the impedance or the flexibility operator (François \& Degrande, 2005; Wolf, 1988), are formulated in the frequency domain. Some other references rely on Laplace formulations of the impedance function (Gaul \& Schanz, 1999; Pereira \& Beer, 2009; Schanz \& Antes, 2006) which allow the use of convolution quadrature methods (Lubich, 1988a).

The approach presented here is essentially an extension of the work of Moser, Antes, and Beer $(2005 \mathrm{a}, 2005 \mathrm{~b})$ which is based on Laplace-domain formulations. This allows us to take advantage of the convolution quadrature method (Lubich, 1994). Since these convolution integrals build the applied forces, the polynomial part of the impedance function can be factorised in order to introduce inertia, damping and stiffness terms typically arising in standard dynamic problems. Hence, this approach combines the underlying principles of some other methods proposed by authors such as Wolf (1995) or Karpel (1982). The problem of the evaluation of the interaction forces is first introduced regardless of the physical nature of the interacting domains. Afterwards, the particular case of dynamic SSI problems will be presented throughout a numerical example.

\section{The hybrid Laplace-time approach}

Let $\Omega_{1}$ be a bounded subset of $\mathbb{R}^{3}$ with a smooth boundary $\partial \Omega_{1}$. Let homogeneous boundary conditions be applied on $\partial \Omega_{1} \backslash \Gamma, \Gamma$ being the interface between the $\Omega_{1}$ and the subdomain $\Omega_{2}$. Let an impedance function $s \mapsto \hat{\mathbf{Z}}(s)$ defined on $\Gamma$ in the Laplace domain be assumed analytic on the complex half-plane $\Re e(s)>\sigma_{0}$ and bounded for large $|s|$ :

$$
\|\hat{\mathbf{Z}}(s)\|_{\mathcal{H}} \leq C\left(\sigma_{0}\right)|s|^{\mu}, \quad \text { with } C\left(\sigma_{0}\right), \mu \in \mathbb{R}
$$

where $s \in \mathbb{C}$ denotes the Laplace variable and $\|\cdot\|_{\mathcal{H}}$ denotes the norm of a proper Hilbert space $\mathcal{H}$. Let its inverse Laplace transform be denoted by $t \mapsto \mathbf{Z}(t), t \in \mathbb{R}$.

In this framework, the problem to be considered is the evaluation of a convolution integral between two causal functions:

$$
(\mathbf{Z} \times \mathbf{u})(t)=\int_{0}^{t} \mathbf{Z}(t-\tau) \mathbf{u}(\tau) \mathrm{d} \tau, \quad 0 \leq t \leq T
$$

where the impedance $\mathbf{Z}(t)$ is the kernel of the convolution and $\mathbf{u}(t)$ denotes a sufficiently time-differentiable function - a causal function is zero for $t<0$. Hereafter, thanks to the existence of an Hilbert basis, fields with respect to space are assumed to be approximated on a finite basis such classically done in the finite element (FE) method.

Let $\hat{\mathbf{Z}}(s)=\hat{\mathbf{Z}}_{m}(s) \hat{\mathbf{P}}(s)$ with $\hat{\mathbf{P}}(s)$ a $s$-polynomial function of order $m \geq \mu>0$ with matrix-valued coefficients of dimension $n_{\Gamma}$, the number of degrees-of-freedom on the interface $\Gamma$ (for $\mu \leq 0$, polynomial $\hat{\mathbf{P}}(s)$ can be chosen as the identity matrix). Following this polynomial decomposition, the distributional convolution kernel $\mathbf{Z}(t)$ is thus written as the product of a sum of $p$-order derivatives $(0 \leq p \leq m)$, which are ponderated by matrix-valued 
coefficients $\quad \boldsymbol{\Lambda}_{p} \in \mathbb{M}_{n_{\Gamma}}^{+}(\mathbb{R})$ and a continuous exponetially bounded function $\mathbf{Z}_{m}(t)=\mathcal{L}^{-1}\left(\hat{\mathbf{Z}}_{m}(s)\right)$, i.e.

$$
\mathbf{Z}(t)=\mathbf{Z}_{m}(t)\left(\sum_{p=0}^{m} \boldsymbol{\Lambda}_{p} \frac{d^{p}}{d t^{p}}\right)
$$

Remark that, by Cauchy's integral theorem, $\mathbf{Z}_{m}(t)$ is a causal function. Thus:

$$
(\mathbf{Z} \times \mathbf{u})(t)=\sum_{p=0}^{m}\left(\int_{0}^{t} \mathbf{Z}_{m}(\tau) \boldsymbol{\Lambda}_{p} \mathbf{u}^{(p)}(t-\tau) d \tau\right)
$$

with $\mathbf{u}(t)$ a smooth causal function.

Therefore, if $\Delta t>0$ denotes the time step, the convolution quadrature method (Lubich, 1988a) approximates the convolution integral in Equation (4) by a discrete convolution as:

$$
(\mathbf{Z} \times \mathbf{u})(n \Delta t)=\sum_{k=1}^{n}\left(\boldsymbol{\psi}_{1}^{n-k+1} \mathbf{u}_{k}+\ldots+\boldsymbol{\psi}_{m}^{n-k+1} \mathbf{u}_{k}^{(m)}\right)
$$

where $\mathbf{u}_{k}^{(p)} \approx \mathbf{u}^{(p)}(k \Delta t)$ and coefficients $\left\{\boldsymbol{\psi}_{j}^{i}\right\} \in \mathbb{M}_{n_{\Gamma}}^{+}(\mathbb{R})(i=1 \ldots n, j=1 \ldots m)$ correspond to the weights of the following generating power series:

$$
\sum_{k=0}^{+\infty} \boldsymbol{\psi}_{\mathbf{j}}^{\mathbf{k}} \zeta^{k}=\hat{\mathbf{Z}}_{m}\left(s_{\Delta t}\right) \Lambda_{j}
$$

The present article addresses the case where the complex variable $s_{\Delta t}$ is given by the rational function of a linear multistep method. In particular, $s_{\Delta t}$ is assumed equal to $\frac{\delta(\zeta)}{\Delta t}$, where $\delta(\zeta)=\frac{3}{2}-2 \zeta+\frac{1}{2} \zeta^{2}$ corresponds to a linear second-order multistep method (the second-order backward differentiation formula or simply BDF-2).

\section{Application to dynamic SSI problems}

The classical dynamic SSI method is based on a domain decomposition technique, where the whole soil-structure domain is decomposed into two subdomains: the soil and the structure. The main reason for this decomposition is that different numerical methods can be used for the soil and for the structure. On the one hand, the bounded subdomain of the structure can be modelled by means of a FE method which allows to take into account non-linear phenomena in a straightforward way. On the other hand, the unbounded linear elastic soil can be computed using a boundary element (BE) method, the radiation conditions being thus implicitly satisfied. When the soil shows a non-linear behaviour, the corresponding part of this subdomain should be incorporated into the structure subdomain. Since engineers are usually more interested in the structural response, it is of interest to directly solve the global problem in the building and to take into account the effect of the soil in the form of a particular type of boundary condition. This condition is expressed by means of the impedance operator, known in the Laplace domain, defined in the previous section. The soil impedance matrix, i.e. the discretised version of the impedance operator, maps any displacement vector of the soil-structure interface to its corresponding force vector on the same boundary. 
When non-linearities are accounted for, the problem have to be formulated in the time domain. Consequently, the interaction forces on the soil-structure interface, denoted again by $\Gamma$, result in a convolution product between the time dynamic soil impedance matrix $t \mapsto \mathbf{Z}(t)$ and the degrees-of-freedom located on the interface $\mathbf{u}_{\Gamma}(t)$ as in Equation (2).

In order to satisfy the condition stated in Equation (1), the computed dynamic soil impedance matrix will be assumed of the following form:

$$
\hat{\mathbf{Z}}(s)=\mathbf{M}_{\Gamma} s^{2}+\mathbf{C}_{\Gamma} s+\mathbf{K}_{\Gamma}+\hat{\mathbf{Z}}_{n s}(s)
$$

where $\mathbf{K}_{\Gamma}, \mathbf{C}_{\Gamma}$ and $\mathbf{M}_{\Gamma}$ are the equivalent mass, damping and stiffness matrices of the soil and where $\hat{\mathbf{Z}}_{n s}(s)$ denotes a non-singular function vanishing for large $|s|$.

Paying attention to the physical units of Equation (2), it seems natural to express the convolution not only in terms of displacements, but also in terms of accelerations and velocities. To that end, the polynomial part of the computed impedance has to be identified and factorised yielding:

$$
\hat{\mathbf{Z}}(s)=\hat{\mathbf{Z}}(s) \hat{\mathbf{P}}^{-1}(s) \hat{\mathbf{P}}(s)=\hat{\mathbf{Z}}(s) \hat{\mathbf{P}}^{-1}(s)\left(\tilde{\mathbf{M}}_{\Gamma} s^{2}+\tilde{\mathbf{C}}_{\Gamma} s+\tilde{\mathbf{K}}_{\Gamma}\right)
$$

where $\tilde{\mathbf{K}}_{\Gamma}, \tilde{\mathbf{C}}_{\Gamma}$ and $\tilde{\mathbf{M}}_{\Gamma}$ are, respectively, the estimators of the matrices $\mathbf{K}_{\Gamma}, \mathbf{C}_{\Gamma}$ and $\mathbf{M}_{\Gamma}$ defined in Equation (7).

Therefore, if $\hat{\mathbf{Z}}_{m}(s)$ denotes $\hat{\mathbf{Z}}(s) \hat{\mathbf{P}}^{-\mathbf{1}}(s)$, the SSI forces can be written in terms of the Laplace transform as follows:

$$
(\mathbf{Z} \times \mathbf{u})(t)=\frac{1}{2 \pi i} \int_{\sigma_{0}+i \mathbb{R}} \hat{\mathbf{Z}}_{m}(s) \hat{\mathbf{P}}(s) \hat{\mathbf{u}}(s) e^{s t} \mathrm{~d} s
$$

Hence, the polynomial $\hat{\mathbf{P}}(s)$ acts over the displacement as a differential operator and Equation (2) finally reads:

$$
(\mathbf{Z} \times \mathbf{u})(t)=\left(\mathbf{Z}_{m} \times \tilde{\mathbf{M}}_{\Gamma} \ddot{\mathbf{u}}\right)(t)+\left(\mathbf{Z}_{m} \times \tilde{\mathbf{C}}_{\Gamma} \dot{\mathbf{u}}\right)(t)+\left(\mathbf{Z}_{m} \times \tilde{\mathbf{K}}_{\Gamma} \mathbf{u}\right)(t)
$$

where vector of interaction forces (denoted hereafter by $\mathbf{R}_{\Gamma}(t)$ ) involves in its calculation, the evaluation of displacement, velocity and acceleration convolutions.

If a time step $\Delta t>0$ is chosen, the convolution integral can be discretised as in Equation (5) leading to:

$$
\mathbf{R}_{\Gamma, n}=\sum_{k=1}^{n}\left(\boldsymbol{\psi}_{2}^{n-k+1} \ddot{\mathbf{u}}_{k}+\boldsymbol{\psi}_{1}^{n-k+1} \dot{\mathbf{u}}_{k}+\boldsymbol{\psi}_{0}^{n-k+1} \mathbf{u}_{k}\right)
$$

where the matrices multiplying the displacement vectors $\mathbf{u}_{k}$, the velocity vectors $\dot{\mathbf{u}}_{k}$ and the acceleration vectors $\ddot{\mathbf{u}}_{k}$ are, respectively, given by:

$$
\begin{aligned}
\boldsymbol{\psi}_{0}^{k} & =\mathbf{Z}_{m}^{k} \tilde{\mathbf{K}}_{\Gamma} \\
\boldsymbol{\psi}_{1}^{k} & =\mathbf{Z}_{m}^{k} \tilde{\mathbf{C}}_{\Gamma} \\
\boldsymbol{\psi}_{2}^{k} & =\mathbf{Z}_{m}^{k} \tilde{\mathbf{M}}_{\Gamma}
\end{aligned}
$$

From a numerical point of view, the true unknown terms in Equation (11) are those at $t=n \Delta t$, since all previous time steps have already been computed. Therefore, instant $n$ can be isolated so that Equation (11) becomes: 


$$
\mathbf{R}_{\Gamma, n}=\boldsymbol{\psi}_{2}^{1} \ddot{\mathbf{u}}_{n}+\boldsymbol{\psi}_{1}^{1} \dot{\mathbf{u}}_{n}+\boldsymbol{\psi}_{0}^{1} \mathbf{u}_{n}+\mathbf{R}_{\Gamma(n-1)}
$$

Coefficients $\psi_{i}^{1}$ and $(i=0,1,2)$ are, respectively, the instantaneous stiffness, damping and inertia terms, whereas $\mathbf{R}_{\Sigma(n-1)}$ is the additional force due to previous time steps:

$$
\mathbf{R}_{\Gamma(n-1)}=\sum_{k=1}^{n-1}\left(\boldsymbol{\psi}_{2}^{n-k+1} \ddot{\mathbf{u}}_{k}+\boldsymbol{\psi}_{1}^{n-k+1} \dot{\mathbf{u}}_{k}+\boldsymbol{\psi}_{0}^{n-k+1} \mathbf{u}_{k}\right)
$$

It is worth noticing that notation $\mathbf{R}_{\Gamma(n-1)}$ has been chosen instead of $\mathbf{R}_{\Gamma, n-1}$ because no recurrence relation exists between the terms of a discrete convolution.

In order to test the hybrid Laplace-time approach for SSI problems, two lumped numerical models are considered. The first one deals with a linear system, whereas the second one shows a material non-linear behaviour.

\subsection{Linear numerical application}

The numerical model considered consists of two masses, $m_{1}$ and $m_{2}$, sketching, respectively, the structure and the foundation. These masses are coupled by a spring of stiffness $k$ and a dashpot $c$ accounting for viscous dissipation in the structure. A rigid body constraint exists between the mass $m_{2}$ and the surface foundation modelled with 2D FEs (see Figure 1(a)). This surface foundation lies over an elastic homogeneous unbounded soil whose impedance matrix is computed using a BE method in the Laplace domain. For the sake of simplicity, a surface foundation having an almost quadratic impedance with respect to the Laplace parameter $s$ is considered so that a time reference solution can straightforwardly be obtained. The numerical values taken for the simulation are listed in Table 1.

A seismic load characterised be the acceleration time history $\gamma(t)$ shown in Figure 1(b) with a maximum acceleration around $.3 \mathrm{~g}$ is applied to $m_{2}$ in the $x$-direction.
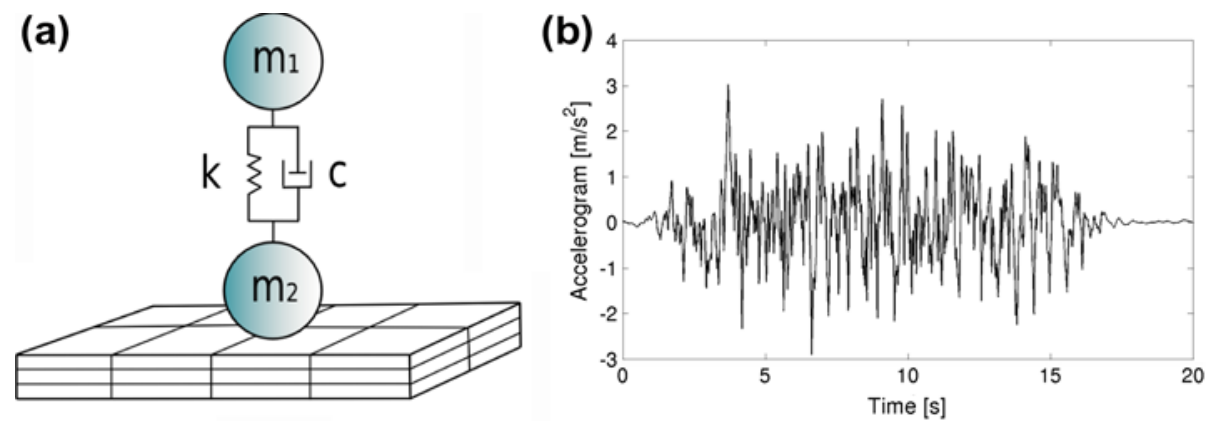

Figure 1. (a) Simplified model of a structure on a square surface foundation. (b) Free-field accelerogram applied to $m_{2}$ as a representation of the seismic loading.

Table 1. Properties of the structure $\left(m_{1}, m_{2}, k\right.$ and $\left.c\right)$ and the soil $(E, \rho$ and $v)$.

\begin{tabular}{lllllll}
\hline$m_{1}(\mathrm{~kg})$ & $m_{2}(\mathrm{~kg})$ & $k\left(\mathrm{~N} \mathrm{~m}^{-1}\right)$ & $c\left(\mathrm{~N} \mathrm{~s} \mathrm{~m}^{-1}\right)$ & $\rho\left(\mathrm{kg} \mathrm{m}^{-3}\right)$ & $E\left(\mathrm{~N} \mathrm{~m}^{-2}\right)$ & $v(-)$ \\
\hline $1.8 \cdot 10^{7}$ & $3.6 \cdot 10^{6}$ & $2.1 \cdot 10^{10}$ & $1.23 \cdot 10^{7}$ & $2.1 \cdot 10^{3}$ & $1.5 \cdot 10^{9}$ & .4 \\
\hline
\end{tabular}


The corresponding linear system, solved in the time domain and using notation of Figure 1 (a), reads:

$$
\left[\begin{array}{cc}
\mathbf{L}_{1}(\cdot) & 0 \\
0 & \mathbf{L}_{2}(\cdot)
\end{array}\right]\left[\begin{array}{l}
\mathbf{u}_{1}(t) \\
\mathbf{u}_{2}(t)
\end{array}\right]+\left[\begin{array}{c}
\mathbf{0} \\
\mathbf{R}_{2}(t)
\end{array}\right]=\left[\begin{array}{c}
\mathbf{0} \\
\mathbf{F}_{2}(t)
\end{array}\right]
$$

where a time differentiation operator $\mathbf{L}_{\lambda}(\cdot)=\mathbf{M}_{\lambda} \partial_{t t}(\cdot)+\mathbf{D}_{\lambda} \partial_{t}(\cdot)+\mathbf{K}_{\lambda}$ for $\lambda \in\{1,2\}$ is introduced. Vector $\mathbf{R}_{2}(t)$ corresponds to the convolution integral of the interaction forces and matrices $\mathbf{M}_{\lambda}, \mathbf{C}_{\lambda}$ and $\mathbf{K}_{\lambda}$ to the inertia, damping and stiffness matrices of the structural discretised model. Solving for the displacement by means of an inconditionally stable Newmark time integration scheme $(\beta=.25, \gamma=.5)$ Equation (15) becomes:

$$
\left[\begin{array}{cc}
\bar{K}_{11} & \mathbf{0} \\
\mathbf{0} & \bar{K}_{22}
\end{array}\right]\left[\begin{array}{c}
c \mathbf{u}_{1, n} \\
\mathbf{u}_{2, n}
\end{array}\right]=\bar{F}_{n}=\bar{F}_{n}-\left\{\begin{array}{c}
\mathbf{0} \\
\mathbf{R}_{\Sigma(n-1)}
\end{array}\right\}
$$

where $\overline{\mathbf{F}}_{n}$ and $\overline{\mathbf{K}}_{i j}(i, j \in\{1,2\})$ denote, respectively, the equivalent vector of forces and the stiffness matrix coming from the application of a Newmark scheme. The term $\overline{\mathbf{K}}_{22}$ actually reads:

$$
\overline{\mathbf{K}}_{22}=\frac{1}{\beta \Delta t^{2}}\left(\mathbf{M}_{22}+\boldsymbol{\psi}_{M}^{1}\right)+\frac{\gamma}{\beta \Delta t}\left(\mathbf{C}_{22}+\boldsymbol{\psi}_{C}^{1}\right)+\left(\mathbf{K}_{22}+\boldsymbol{\psi}_{K}^{1}\right)
$$

where $\boldsymbol{\psi}_{M}^{1}=\mathbf{Z}_{m}^{1} \tilde{\mathbf{M}}_{\text {sol }}, \boldsymbol{\psi}_{C}^{1}=\mathbf{Z}_{m}^{1} \tilde{\mathbf{C}}_{\text {sol }}$ and $\boldsymbol{\psi}_{K}^{1}=\mathbf{Z}_{m}^{1} \tilde{\mathbf{K}}_{\text {sol }}$ come from the impedance matrix of the soil subdomain. Values $\tilde{\mathbf{K}}_{\text {sol }}, \tilde{\mathbf{C}}_{\text {sol }}$ and $\tilde{\mathbf{M}}_{\text {sol }}$ (or $\tilde{\mathbf{K}}_{\Gamma}, \tilde{\mathbf{C}}_{\Gamma}, \tilde{\mathbf{M}}_{\Gamma}$ following notation of Equation (8) are estimated by means of a first frequency computation of the soil impedance matrix, without the non-singular term of Equation (7) and for $s=j \omega(\omega \in \mathbb{R}$ denotes here the angular frequency). Coefficients $\mathbf{Z}_{m}^{k}(k=1 \ldots n)$ can be efficiently computed using Fast Fourier Transforms (FFT) (Lubich, 1988b):

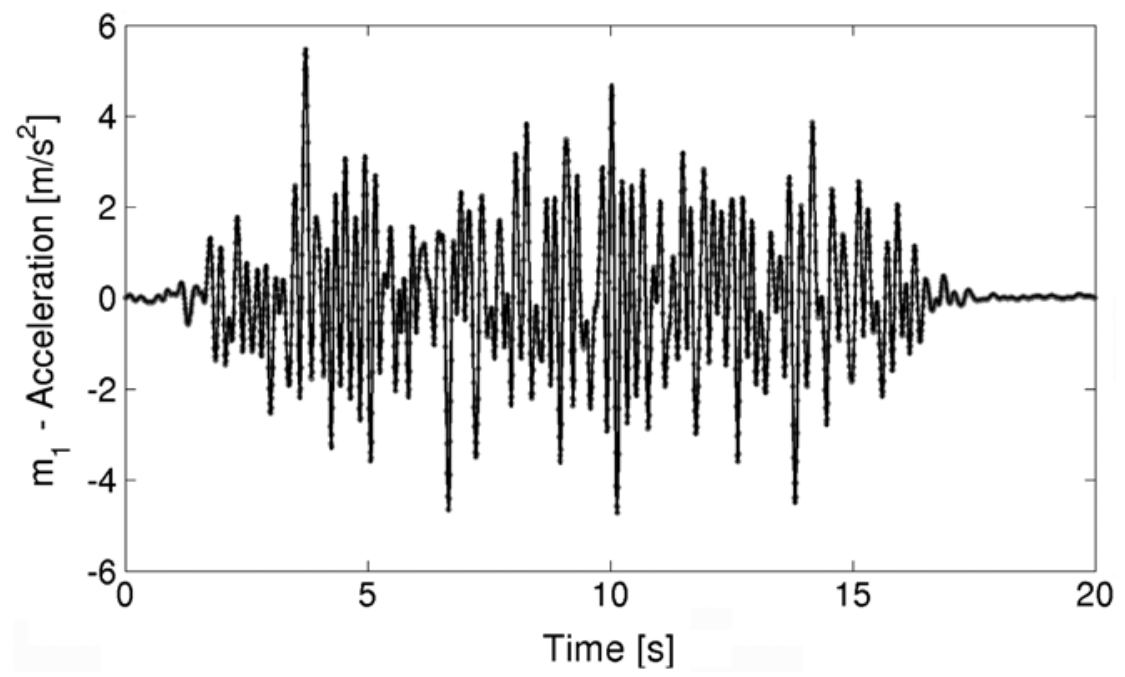

Figure 2. Displacement at $m_{1}$ in the $x$-direction computed with the hybrid Laplace-time domain approach (black line) and compared to the reference solution (grey markers). 


$$
\mathbf{Z}_{m}^{k}=\frac{\rho^{-n}}{L} \sum_{l=0}^{L-1} \hat{\mathbf{Z}}_{m}\left(s_{l}\right) e^{-\frac{2 \pi i n l}{L}}, n=0,1, \ldots, N
$$

where $\rho$ represents the radius of a circle in the analyticity domain of $\hat{\mathbf{Z}}_{m}(s)$ and $s_{l}=\frac{\delta\left(\rho e^{2 \pi i l / L}\right)}{\Delta t}$ with $\delta(\zeta)$ the polynomial of the underlying linear multistep method. Assuming that the values of $\hat{\mathbf{Z}}_{m}$ are computed with precision $\epsilon_{\mathrm{CQM}}$, one gets that the error in $\mathbf{Z}_{m}^{k}$ is $O\left(\sqrt{\epsilon_{\mathrm{CQM}}}\right)$ when $L=N$ and $\rho^{N}=\sqrt{\epsilon_{\mathrm{CQM}}}$. In addition, the FFT algorithm allows to compute the weights in $O(L \log L)$ operations.

It is important to notice that the terms $\psi_{M}^{1}, \psi_{C}^{1}$ and $\psi_{K}^{1}$ in Equation (17) modify the condition number of the equivalent stiffness matrix $\overline{\mathbf{K}}$ and, therefore, their values have thus an impact on the convergence of the solution. Moreover, and contrary to the Newmark forces $\bar{F}_{n}$ which have to be updated at each time step, the Newmark operator $\overline{\mathbf{K}}$ is constant and can be computed once for all.

Figure 2 shows the acceleration of $m_{1}$ computed using the hybrid Laplace-time approach (involving only displacement convolution) compared to a reference solution. A time step of $.01 \mathrm{~s}$ and a precision of $\epsilon_{C Q M}=10^{-10}$ for the convolution quadrature method, which give sufficiently accurate results, have been used. The root mean square error over the maximal acceleration in a $20 \mathrm{~s}$-interval regarding a reference solution is about $\epsilon_{20}=.53 \%$. It is observed that better results are obtained when the polynomial part of impedance is factorised. Indeed, a decomposition involving only inertial terms gives an error of $1.35 \%$, whereas accounting also for stiffness terms reduces the error to $.50 \%$. The error drops to $.43 \%$ when accounting for damping terms in the convolution.

\subsection{Non-linear numerical application}

The non-linear behaviour is introduced by an elastoplastic spring having one end attached to the upper mass $m_{1}$ and the other end attached to a smaller mass $m_{2}$. Analogously to the linear numerical application, a square surface foundation layering on a homogeneous half-space is connected to mass $m_{2}$ by means of a rigid body constraint (see Figure 3(a)) and the loading of Figure 1(b) is considered. The soil impedance seen from the foundation is again computed with a BE method in the Laplace domain. The same properties are considered for the structure and the soil, except that no viscous dissipation is considered in this case. The elastoplastic behaviour of the spring is modelled by means of a linear kinematic work hardening law
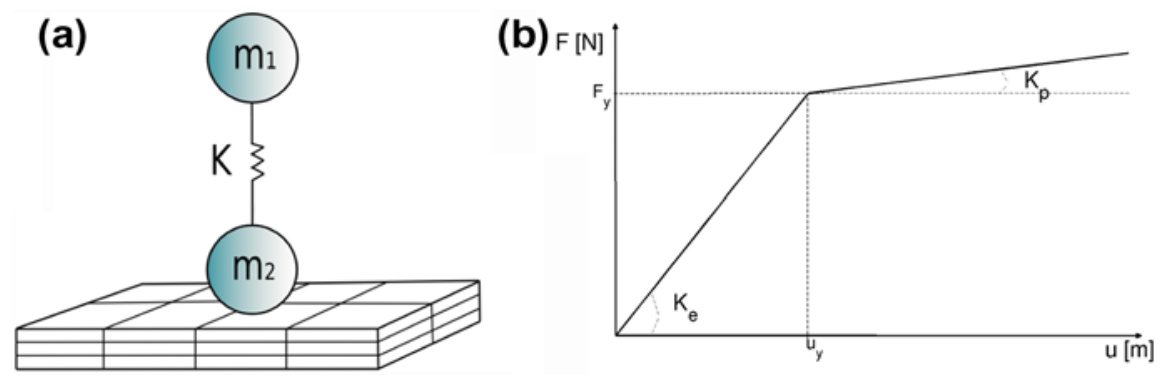

Figure 3. (a) Simplified model of a structure on a square surface foundation. (b) Linear kinematic work hardening law of the non-linear spring $K$. 
sketched in Figure 3(b). The elastic deformation is characterised here by the elastic stiffness $K_{e}=2.1 \times 10^{10}$ N.m which, after reaching the plasticity yield force $F_{y}$, drops to $K_{p}=.1 K_{e}$.

The governing equations of this numerical model from the non-inertial frame of reference of the structure can be written at $t=n \Delta t$ as follows:

$$
\left[\begin{array}{cc}
\mathbf{M}_{1} & \mathbf{0} \\
\mathbf{0} & \mathbf{M}_{2}+\boldsymbol{\psi}_{M}^{1}
\end{array}\right]\left[\begin{array}{l}
\ddot{\mathbf{u}}_{1, n} \\
\ddot{\mathbf{u}}_{2, n}
\end{array}\right]+\left[\begin{array}{c}
\mathbf{F}_{1, n}^{\text {int }} \\
\mathbf{F}_{2, n}^{\text {int }}
\end{array}\right]=\left[\begin{array}{l}
-\mathbf{M}_{1} \mathbf{e}_{x} \gamma_{n} \\
-\mathbf{M}_{2} \mathbf{e}_{x} \gamma_{n}
\end{array}\right]+\left[\begin{array}{c}
\mathbf{0} \\
-\mathbf{R}_{\Sigma(n-1)}
\end{array}\right]
$$

where $\mathbf{F}_{j}^{\text {int }}(t)[j \in\{1,2\})$ denotes the non-linear internal efforts in the structure and depend on both displacement and velocity vectors. The interaction forces $\mathbf{R}_{2, n}$ have been directly substituted by Equation (13). It has to be noticed that the application considered here is particularised to the case where $\tilde{\mathbf{K}}_{\text {sol }}=\tilde{\mathbf{C}}_{\text {sol }}=\mathbf{0}$, that is, the case where only inertial terms are taken into account for the computation of the convolution integral:

$$
\mathbf{R}_{2, n}=\sum_{k=1}^{n} \boldsymbol{\psi}_{M}^{n-k+1} \ddot{\mathbf{u}}_{2, k}=\boldsymbol{\psi}_{M}^{1} \ddot{\mathbf{u}}_{2, n}+\sum_{k=1}^{n-1} \boldsymbol{\psi}_{M}^{n-k+1} \ddot{\mathbf{u}}_{2, k}
$$

where $\boldsymbol{\psi}_{M}^{k}=\mathbf{Z}_{m}^{k} \tilde{\mathbf{M}}_{\text {sol }}$. Compared to the linear case, the account of inertial terms only in the convolution is expected to give higher errors. However, its implementation is easier. Remark that, otherwise, not only the mass of the structure, but also the tangent operator of the non-linear analysis should be modified with the corresponding $\psi_{j}^{k}$ for $j \in\{M, K, C\}$.

The governing equations are finally solved for the displacement vector by using the modified average acceleration time integration scheme of the Newmark family. This time integration scheme allows to introduce numerical damping by means of a parameter denoted here by $\alpha$. For a given plasticity yield force $F_{y}$, previous work showed (Nieto Ferro, Clouteau, Greffet, \& Devésa, 2011) that better agreements with the reference solution are obtained for a

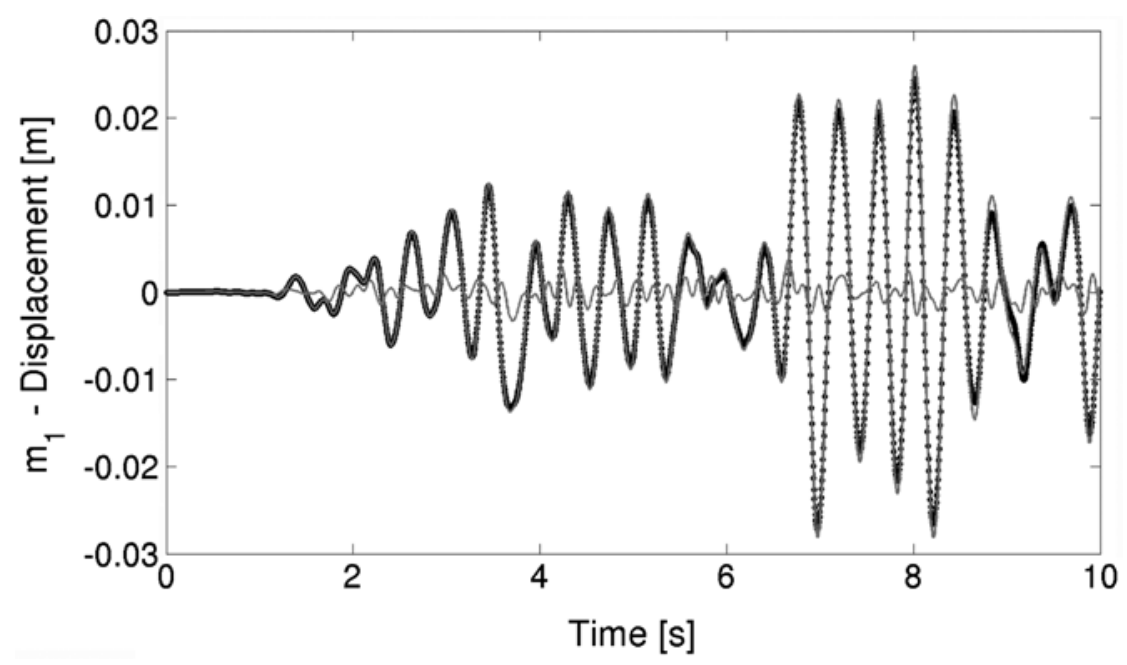

Figure 4. Displacement at $m_{1}$ in the $x$-direction computed with the hybrid Laplace-time domain approach (black markers) and compared to the reference solution (high amplitude grey line) and to the linear response (small amplitude grey line). 
precision of $\epsilon_{\mathrm{CQM}}=10^{-6}$. In addition, it seemed that the more non-linear the response, the larger the error. The latter value of $\epsilon_{\mathrm{CQM}}$ and a time step of $\Delta t=.01 \mathrm{~s}$ have been used for this application.

If the plasticity yield is chosen sufficiently large, the entire model remains linear. Therefore, linear and non-linear responses can easily be compared using the same precision $\epsilon_{\mathrm{CQM}}=10^{-6}$. Figure 4 shows thus the displacement at $m_{1}$ in the $x$-direction compared to the reference solution and also to the linear solution for $F_{y}=8.0 \cdot 10^{6} \mathrm{~N}$. It is then observed that the amplitude of displacements is increased which illustrates indeed the influence of non-linear phenomena.

Further work has to be done in order to investigate if the numerical response is improved, as for the linear application, when the damping and stiffness parts of the dynamic impedance are also factorised.

\section{Conclusions}

The SSI problem is solved directly in the building and the impedance operator, defined on the boundary, rigourously models the unbounded soil. When non-linearities are taken into account, the problem is solved in the time domain. Therefore, the influence of the soil is accounted for as a load (interaction forces) computed as a convolution integral in the time domain.

The proposed approach based on the Laplace domain presents some interesting features. On the one hand, it can be combined with IFFT algorithms yielding to small computational costs. On the other hand, it allows to express the convolution integral in terms of inertial, damping and stiffness quantities.

Two numerical applications have been considered: one being linear and the other exhibiting a non-linear behaviour. In the first case, it has been shown that factorising the soil impedance matrix in order to introduce soil inertial, damping and stiffness terms in the time integration scheme yields to smaller errors compared to a reference solution. In the second case, the convolution integral has only been transformed to a convolution depending only on acceleration quantities. Very satisfactory results have been obtained when compared to a reference solution. However, it seems that the numerical damping introduced by the proposed approach increases when strong elastoplastic behaviour is taken into account. Additional work is thus necessary in order to extend this hybrid Laplace-time domain approach to some industrial numerical models involving, for instance, embedded flexible foundations.

\section{Acknowledgements}

This work has been funded by Électricite de France (EDF), the LaMSID and the French ANRT. The first author gratefully acknowledges their support as well as the supervision of École Centrale Paris.

\section{References}

Cottereau, R., Clouteau, D., Soize, C., \& Cambier, S. (2006). Probabilistic nonparametric models of impedance matrices. Application to the seismic design of a structure. European Journal of Computational Mechanics, 15(1-3), 131-142.

Darbre, G., \& Wolf, J. (1988). Criterion of stability and implementation issues of hybrid frequency time domain procedure for non-linear dynamic analysis. Earthquake Engineering and Structural Dynamics, 16(4), 569-581.

François, S., \& Degrande G. (2005). A time domain coupled boundary element-finite element method for the dynamic response of structures. In J.L. Bento Coelho (Ed.), Proceedings of the 12th International Congress on Sound and Vibration, July 2005, Lisbon, Portugal. 
Gaul, L., \& Schanz, M. (1999). A comparative study of three boundary element approaches to calculate the transient response of viscoelastic solids with unbounded domains. Computer Methods in Applied Mechanics and Engineering, 179, 111-123.

Karpel, M. (1982). Design for active flutter suppression and gust alleviating using state-space unsteady aeroelastic modelling. Journal of Aircraft, 19(3), 221-227.

Lubich, C. (1988a). Convolution quadrature and discretized operational calculus I. Numerische Mathematik, 52, 129-145.

Lubich, C. (1988b). Convolution quadrature and discretized operational calculus II. Numerische Mathematik, 52, 413-425.

Lubich, C. (1994). On the multistep time discretization of linear initial-boundary value problems and their boundary integral equations. Numerische Mathematik, 67, 365-389.

Moser, W., Antes, H., \& Beer, G. (2005a). A Duhamel integral based approach to one-dimensional wave propagation analysis in layered media. Computational Mechanics, 35, 115-126.

Moser, W., Antes, H., \& Beer, G. (2005b). Soil-structure interaction and wave propagation problems in $2 \mathrm{D}$ by a Duhamel integral based approach and the convolution quadrature method. Computational Mechanics, 36, 431-443.

Nieto Ferro, A., Clouteau, D., Greffet, N., \& Devésa, G. (2011). Hybrid Laplace-time domain approach for nonlinear dynamic soil-structure interaction problems. In M. Papadrakakis, M. Fragiadakis, \& V. Plevris (Eds.), Proceedings of the 3rd ECCOMAS thematic conference on computational methods in structural dynamics and earthquake engineering, May 2011, Corfu, Greece.

Pereira, A., \& Beer, G. (2009). Interface dynamic stiffness matrix approach for three-dimensional transient multi-region boundary element analysis. International Journal for Numerical Methods in Engineering, 80, 1463-1495.

Schanz, M., \& Antes, H. (2006). Application of 'Operational Quadrature Methods' in time domain boundary element methods. Meccanica, 32, 179-186.

Wolf, J. (1995). Unit-impulse response matrix of unbounded medium by infinitesimal finite-element cell method. Computer Methods in Applied Mechanics and Engineering, 122, 251-272.

Wolf, J. (1988). Soil-structure interaction analysis in the time domain. Englewood Cliffs, NJ: PrenticeHall. 